\title{
Reply to: doi:10.1007/s00464-013-3395-4: Re: "Laparoscopic bile duct exploration via choledochotomy followed by primary duct closure is feasible and safe..." (2013 (27):4164-4170)
}

\author{
Yazan S. Khaled • Basil J. Ammori
}

Received: 2 January 2014/ Accepted: 20 February 2014/Published online: 28 March 2014

(C) Springer Science+Business Media New York 2014

To the editor,

We agree that it is best to avoid laparoscopic common bile duct exploration (LCBDE) in patients with small diameter bile ducts (amongst our 120 patients who underwent LCBDE, one had a diameter of $3 \mathrm{~mm}, 3$ of $4 \mathrm{~mm}$ and 4 of $5 \mathrm{~mm}$ ). However, exploration of these is possible with the use of a 3-mm choledochoscope or via a trans-cystic duct approach. Whilst single-stage LCBDE is not the preferable option in high-risk patients, at times it becomes unavoidable when the endoscopic approach to management of ductal stones has failed.

In our experience, LCBDE with primary closure can be performed in both elective and emergency cases. We performed emergency LCBDE in ten patients and encountered a postoperative chest infection in one and urinary tract infection in another but no bile leaks or duct strictures. Clearly though, patients with cholangitis, deep jaundice and coagulopathy should be optimised preoperatively and consideration should be given to preoperative percutaneous transhepatic biliary drainage as a temporising measure before embarking on LCBDE.

With the use of a transverse choledochotomy and interrupted 4-0 Vicryl suture closure of non-dilated bile ducts $(<6 \mathrm{~mm}$ diameter $)$ at $\operatorname{LCBDE}(n=8)$, we have encountered no postoperative bile leaks or bile duct strictures.

We agree that endoscopic retrograde cholangiopancreatography (ERCP) and endoscopic nasobiliary drainage have their application in the management of bile leak due to retained stones after LCBDE. However, re-laparoscopy has its role in patients with generalised abdominal signs and systemic disturbance as it facilitates a thorough abdominal washout as well as removal of retained duct stones with re-suturing of the bile duct. Nasobiliary drainage is not commonly adopted in the United Kingdom.

Y. S. Khaled · B. J. Ammori ( $₫)$

Department of Hepato-Pancreato-Biliary Surgery, North

Manchester General Hospital, Manchester, UK

e-mail: bammori@btinternet.com

Y. S. Khaled · B. J. Ammori

University of Manchester, Manchester, UK 